\title{
Influence of the Solidity Ratio on the Small Wind Turbine Aerodynamics
}

\author{
Karol Zawadzki ${ }^{*}$, Wojciech Śmiechowicz ${ }^{1}$, Małgorzata Stępień ${ }^{1}$, Anna Baszczyńska ${ }^{1}$, Michał Tarkowski ${ }^{1}$ \\ ${ }^{1}$ Institute of Turbomachinery, Lodz University of Technology, Stefana Żeromskiego 116, 90-924 Łódź, Poland
}

\begin{abstract}
Increasing popularity of individualised electricity generation from wind by prosumers creates a strong demand for profitable and highly efficient small wind turbines. This paper investigates the influence of rotor blade solidity parameter on device efficiency in hope of determining its optimal value as a part of the development process of the GUST small wind turbine. The study involved experimental analysis in the wind tunnel and numerical simulations performed in QBlade software. Different solidities of the rotor were achieved by alteration of (1) number of blades and (2) chord distribution along the blade span. The increase of rotor solidity resulted in augmentation of the aerodynamic efficiency in both approaches. The elongation of the chord by $33 \%$ in a 3-bladed rotor resulted in a bigger power coefficient increment than addition of a $4^{\text {th }}$ blade with original chord distribution. Even though the solidity was the same, the 3-bladed rotor performed better, possibly due to lower form drag. The results emphasize the importance of the rotor solidity optimization during the small wind turbine rotor development and may significantly influence overall power output.
\end{abstract}

\section{Introduction}

In view of the European Union's plans for achieving climate neutrality by 2050 , there is a growing concern regarding the reduction of greenhouse gas emission. This target can be attained by switching to renewable energy sources like wind energy. In parallel to an increasing number of offshore- and onshore wind farms, a new market has recently emerged: electricity generation by individual household owners, called prosumers. They utilize wind turbines of much smaller diameter than the industrial-grade machines in order to satisfy domestic needs. Still, small wind turbines (SWTs) have to be profitable. To achieve that, the blade geometry has to be optimized to provide high efficiency. The increasing popularity of small wind turbines provoked the growth of methods allowing aerodynamic performance analysis and development of these devices.

One of the most common methods used to assess the aerodynamic performance of wind turbine is the Blade Element Momentum (BEM) theory. It is a simple and highly efficient method used to predict wind turbine performance. Despite its limitations associated with dependence on the quality of the 2D aerofoil data, it is extensively used by researchers for wind turbine blade preliminary design [1]. Muhsen et al. [2] used the BEMbased Qblade algorithm to design and optimise the blades for a small wind turbine rotor. The aerofoils S1210 and S1223 were used to construct a three-bladed rotor of diameter equal to $4 \mathrm{~m}$. At the wind speed of $7 \mathrm{~m} / \mathrm{s}$, the wind turbine was able to generate $1.18 \mathrm{~kW}$, reaching the power coefficient equal to 0.40 . The Qblade software was also used by Suresh and Rajakumar [3] in the design process of a small-scale horizontal axis wind turbine (HAWT) for rural applications. The authors aimed to design a $2 \mathrm{~kW}$ HAWT with $1.8 \mathrm{~m}$ rotor diameter, using the SD7080 aerofoil, suitable for low wind speed applications.

On the other hand, experimental analyses offer usually a very good quality and repetitiveness of the results in a short period of time, if the infrastructure is available to the researcher. Lipian et al. [4] performed wind tunnel tests of a scaled wind turbine in shroudedand twin-rotor systems. They found out that the shroud around the rotor increased its efficiency much more than an addition of a second rotor in the wake, owing to a bigger mass flow rate. Kulak et al. [5] analysed experimentally small wind turbine blades equipped with winglets at the blade tips to prevent tip losses. The authors indicated that in low Reynolds number (Re) regime, the winglets at the blade tip can increase the overall power output.

The geometry of a wind turbine blade, constructed basing on the selected aerofoil, is one of the key factors determining wind turbine performance. The parameters defining blade geometry are the chord length distribution $\mathrm{c}$ and the blade twist [6]. The number of blades $\mathrm{N}$ and the chord distribution have a direct influence on the blade solidity $\sigma$ which is defined as a ratio of the rotor planform area to the rotor swept area A. Solidity at the blade local radius $\mathrm{r}$ is described by the following mathematical formula [7]:

$$
\sigma(r)=c(r) N / A
$$

\footnotetext{
* Corresponding author: 238549@edu.p.lodz.pl
} 
Given constant rotor swept area, the solidity can be altered by changing the number of blades and/or chord modification. As presented in [7], low solidity rotors generate low torque, but operate at higher rotational velocities. On the other hand, if solidity is high, rotor can generate bigger torque at lower rotor speed. This dependency is presented in Fig. 1, which shows the influence of the number of blades on the rotor power coefficient as a function of the tip speed ratio (TSR) of a rotor of particular chord distribution and swept area. It can be observed that solidity has a great influence on both the rotor maximum power coefficient and the operational range of the tip speed ratio.

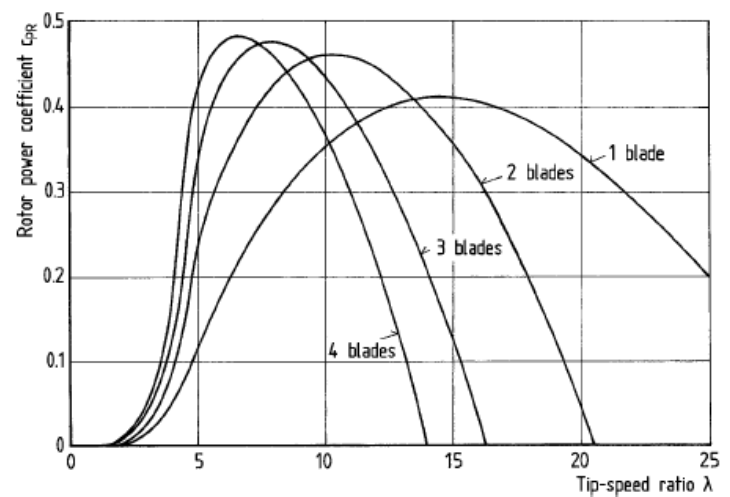

Fig. 1. Influence of number of blades on wind turbine power coefficient [6]

The aim of this research is to investigate the influence of the rotor solidity on the performance of a GUST small horizontal axis wind turbine. The solidity was modified by the increase of the number of blades and chord distribution. Firstly, the experimental analysis performed for scaled rotors in a wind tunnel located at the Institute of Turbomachinery at Lodz University of Technology (IMP TUL) was performed. Later, the experimental data was compared with outcomes of numerical analysis performed in Qblade software.

\section{Methodology}

The performed analysis combined experimental and numerical assessment methods. Rotors operating with different values of solidity were simulated with QBlade software, which utilizes BEM scripts. The classical BEM theory integrates results of the actuator disc assumptions, which describe physical parameters around the rotor in general, with a discretized system of equations. Main calculations are executed for a rotor model with an infinite number of blades. Real parameters, such as an actual blade number and their geometrical characteristics, are employed as mathematical corrections in the formulas [8].

The experimental campaign was conducted in the open-section wind tunnel facility at IMP TUL. The circular outlet of the wind tunnel has a diameter of $0.8 \mathrm{~m}$, allowing to test the prototype model in 1:4 scale (scaled rotor diameter equal to $0.4 \mathrm{~m}$ ). Resultant blockage ratio did not exceed $25 \%$, remaining in an acceptable range for the open-jet wind tunnel examinations of small wind turbines [9].

The measurement series presented in the paper were performed for the same constant wind speed of $12 \mathrm{~m} / \mathrm{s}$. The tests aimed at collecting power coefficient versus tip speed ratio characteristics of rotors with different solidity values. In the first measurement series the blade geometry was preserved, but their number was altered (2, 3 and 4 blades mounted on the hub), affecting rotor's solidity. In the second measurement session, the threebladed rotor was equipped with blades of chord lengths extended by $33 \%$, comparing to the default ones. The three-bladed rotor with enlarged chords represented the same solidity as the four-bladed rotor with default chord lengths.

All sets of examined blades, as well as the hub, were produced with the Fused Deposition Modelling (FDM) 3D printing technology. During the tests, the hub was additionally colvered with a dome casing, which was also 3D printed. Utilization of 3D printing technology in wind tunnel testing allows to significantly reduce time required for model manufacturing [10].

The test stand (visible in Fig. 2) was placed downstream the wind tunnel outlet. The rotor plane of rotation was at a distance of $1 \mathrm{~m}$ from the wind tunnel outlet. The hub with exchangeable sets of blades (1) was mounted on a shaft which was supported by a bearing unit (2) and connected to the torque meter (3) and generator (4).

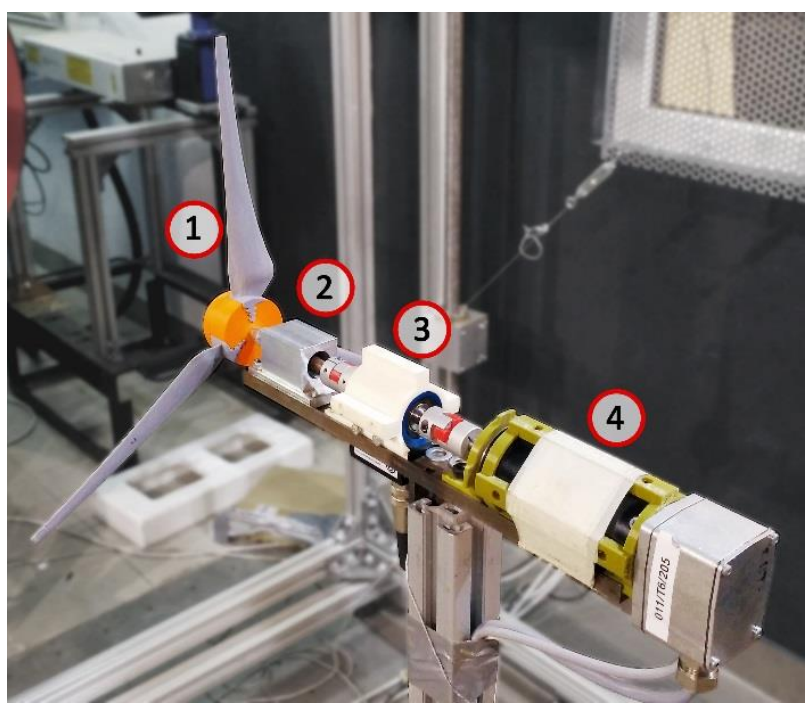

Fig. 2. Open-jet wind tunnel test stand for small wind turbine analysis (1 - 3D-printed rotor model; 2 - bearing unit; 3 torque meter; 4 - motor/generator device)

Measurement of wind turbine rotational speed was provided. The electrical circuit of the test bench was connected with a group of batteries, with current and voltage meters attached for monitoring. The wind tunnel test section was equipped with atmospheric pressure, temperature and relative humidity sensors. The Prandtltype Pitot tube was placed at the tunnel outlet, to assess wind velocity. The data was collected by a digital acquisition system as 32768 samples, with acquisition 
rate equal to $1 \mathrm{kHz}$, which is a standard procedure for IMP TUL laboratory of aerodynamics [4].

The measurements were conducted at several fixed wind speeds. The rotational velocity of the rotor was increased with a certain step, resulting in different values of tip speed ratio. Control of the wind turbine was performed by a motor/generator device, powered from the circuit batteries. The same set of batteries was used as storage for energy produced by the tested turbine. Wind velocity in the test section was determined using differential pressure measurements. The mechanical power output of the wind turbine was obtained throughout measurements of the rotor torque and rotational speed, allowing to determine power and power coefficient value.

\section{Experimental and numerical analysis}

In this chapter, the outcomes of numerical simulations and wind tunnel experiments will be illustrated. Five configurations of the rotor were investigated. First three rotor variants (2-, 3- and 4-bladed) were equipped with blades possessing the default chord length $(0.02 \mathrm{~m}$, marked as " 1.00 "). The chord lengths of two remaining rotor versions (3- and 4-bladed) were extended by $33 \%$ (being equal to $0.0267 \mathrm{~m}$, marked as " 1.33 "). The summary of all variants tested experimentally or numerically (or both) is presented in Table 1 .

Table 1. Summary of all investigated variants

\begin{tabular}{|c|c|c|}
\hline $\begin{array}{c}\text { Number of } \\
\text { blades }\end{array}$ & $\begin{array}{c}\text { Chord } \\
\text { length }\end{array}$ & Type of the test \\
\hline 2 & 1.00 & Experimental \\
\hline 3 & 1.00 & $\begin{array}{c}\text { Experimental, } \\
\text { numerical }\end{array}$ \\
\hline 3 & 1.33 & $\begin{array}{c}\text { Experimental, } \\
\text { numerical }\end{array}$ \\
\hline 4 & 1.00 & $\begin{array}{c}\text { Experimental, } \\
\text { numerical }\end{array}$ \\
\hline 4 & 1.33 & Numerical \\
\hline
\end{tabular}

It was decided to perform all tests at the wind speed $12 \mathrm{~m} / \mathrm{s}$, so as to satisfy the similarity criterion between the scaled-down model and the original rotor. Furthermore, wind speed higher than $12 \mathrm{~m} / \mathrm{s}$ could create excessive aerodynamic forces, resulting in destruction of 3D-printed blades.

First two Subsections of this Section are devoted to the experimental and numerical investigation of the blade with default chord length ("1.00"), while the last Subsection aims to discuss the influence of the elongated chord ("1.33").

\subsection{Experimental tests}

First session of tests was performed to compare the performance of 2-, 3- and 4-bladed rotor with the default chord length ("1.00").

Fig. 3 presents the dependence of power coefficient (aerodynamic efficiency, cp) on different tip speed ratio values. The characteristics aim to compare the power efficiency between a rotor with 2, 3 and 4 blades. The rotor with 4 blades achieves cp values as high as 0.27 , which is remarkably bigger (by approximately 23\%) than maximum $\mathrm{cp}$ for a 3-bladed rotor (0.22). Both configurations reach the maximal power coefficient at TSR around 4.5, with a negligible shift towards higher TSR values in case of a version with 3 blades.

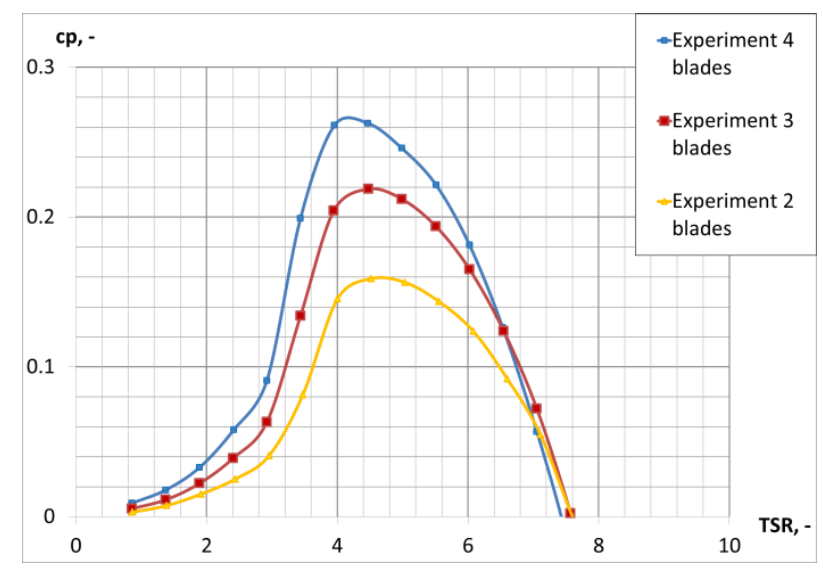

Fig. 3. The power coefficient as a function of the tip speed ratio for different number of blades at $12 \mathrm{~m} / \mathrm{s}$

However, for the highest TSR values (over 6.5), the 3-bladed rotor attains higher cp magnitudes than the 4bladed variant, which stands in opposition to outcomes at lower TSR values. Regarding the 2-bladed rotor, it achieves an overall smaller cp coefficient, with maximal value equal to 0.16 for $\mathrm{TSR}=4.5$. These observations follow the previous predictions: greater number of blades results in a greater torque, which has a direct impact on the achieved power and lowers the cut-in wind speed of the rotor. This phenomenon can be beneficial notably in case of small wind turbines. On the other hand, the remarkably higher aerodynamic efficiency of the 4-bladed rotor indicates that there is a great room for possible improvements of the default geometry (3-bladed rotor) properties.

For the highest TSR values $(>7)$ the influence of drag reduction can be observed: the 2-bladed rotor is characterized by higher $\mathrm{cp}$ values than a 4-bladed one.

\subsection{Numerical simulations}

Identical aerodynamic conditions (wind speed $12 \mathrm{~m} / \mathrm{s}$, rotor scale 1:4) were imposed in the QBlade simulations. Two most efficient types of rotor configuration ( 3 and 4 blades) were investigated numerically, and results were compared with the experimental data. Simulations were performed with Ncrit parameter equal to 9, following the recommendation of Drela [11]. Plots of $\mathrm{cp}(\mathrm{TSR})$ for experimental and numerical results are shown in Fig. 4. 


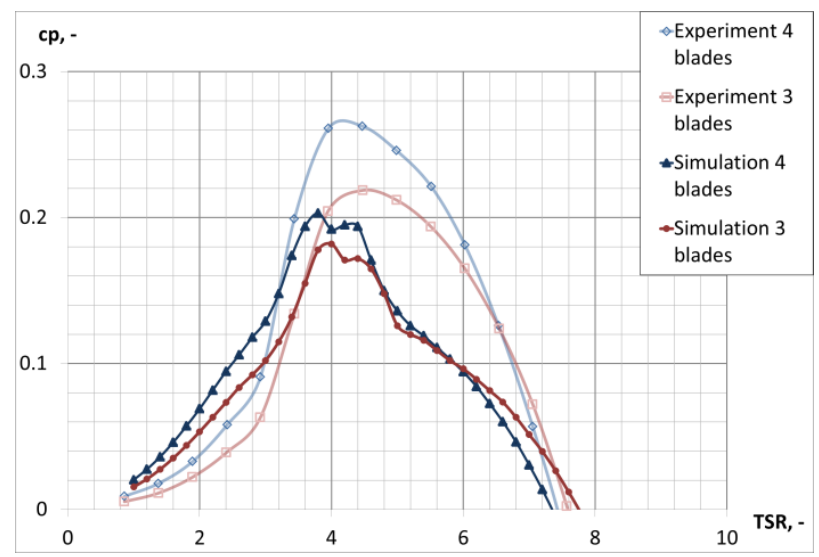

Fig. 4. Comparison of power curves from simulation and wind tunnel tests at $12 \mathrm{~m} / \mathrm{s}$

Noticeable similarities can be observed for the simulation datasets. The 4-bladed rotor attains higher $\mathrm{cp}$ value than the 3 -bladed rotor $(0.20$ vs. 0.18 , respectively), which mimics a similar tendency as in case of aerodynamic experiments. Additionally, in case of both tests (experimental and numerical) the rotor of lower solidity (3-bladed) operates in a slightly wider range of TSR than the 4-bladed one. This is consistent with theoretical predictions mentioned in Section 1.

Some significant discrepancies between simulation and experiment can be spotted. The greatest difference is that in both ( 3 and 4 blades) cases, the simulation predicts a considerably lower power coefficient values (cp) than the experiment reveals. Furthermore, according to simulation data, the optimal tip speed ratio value is equal to 3.8 , which differs from the wind tunnel test outcomes (optimal TSR $>4$ ). The power coefficient values obtained from the wind tunnel experiment were relatively greater than those predicted by the Qblade software. The reason behind this difference may be the theoretical instability and transitional nature of the flow in case of a scaled-down rotor. This phenomenon was indeed reproduced by the QBlade results. However, during the wind tunnel tests, the actual level of turbulence intensity was higher than the simulation predicted, which tends to be beneficial from the efficiency point of view in the considered Reynolds number range. Hence, the values of $\mathrm{cp}$ max are higher in case of the wind tunnel tests, comparing to the QBlade simulation results.

\subsection{Investigation of the chord length}

As mentioned in Section 1, the solidity ratio depends on the number of blades and chord distribution along the blade. Theoretically, increasing the chord by $1 / 3$ has the same impact on solidity ratio, as the change of the number of blades from 3 to 4 . As mentioned earlier, the average chord of the original (reference) blade was equal to $0.02 \mathrm{~m}$, therefore the average chord increased by $33 \%$ is equal to approx. $0.0267 \mathrm{~m}$. Numerical simulations and wind tunnel tests were performed to check the aerodynamic performance of a rotor with blades with enlarged chord (being a 1.33 of a reference chord).
Fig. 5 allows to compare the maximal power coefficient and TSR range of a rotor having blades with the default chord (marked as "1.00"), with the version of a modified chord (marked as "1.33"). From the plot it can be noticed that during the experiment, the " 3 blades 1.33 " configuration attains the highest power efficiency among all other variants tested in the wind tunnel $(\mathrm{cp}=$ 0.29 , comparing with 0.27 and 0.22 for 4 -bladed 1.00 and 3-bladed 1.00 versions, respectively). Moreover, it operates at the widest range of TSR, up to 8. However, better performance of an increased-chord rotor can be somehow related to the aerofoil geometry reproduction quality. Blades having the chord increased by $1 / 3$ (comparing to the original variant) are less prone to the manufacturing method-resulting imperfections, and the blade is also more stiff and less prone to bending in operation.

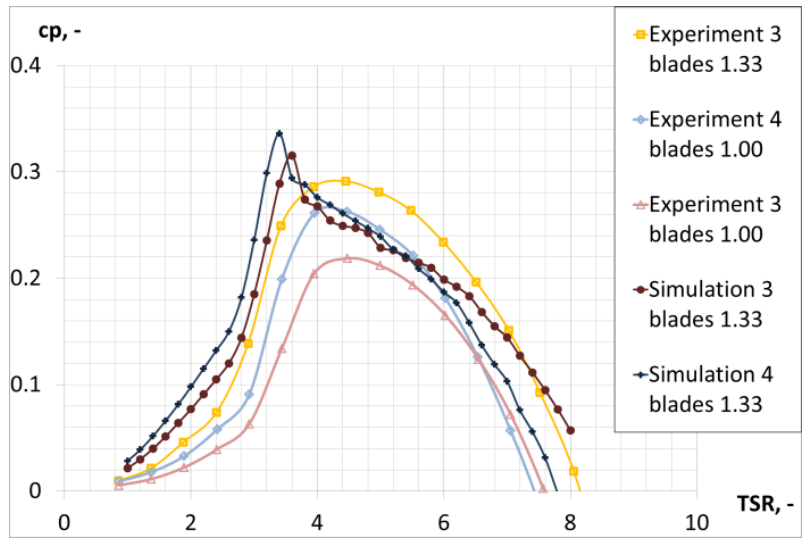

Fig. 5. Comparison of the power coefficient $\mathrm{cp}$ for different blade chord at $12 \mathrm{~m} / \mathrm{s}$

Numerical simulations of the power curves predict a significant rise of the maximal power coefficient value with the solidity increase. In the graph presented in Fig. 4, the outcomes of numerical simulations of original "blades $1.00 "$ for $12 \mathrm{~m} / \mathrm{s}$ are presented. Comparing them with results of "blades 1.33 " in Fig. 5, one can point out the significant difference in cp max value - about 0.14 for both variants (3- and 4-bladed).

Summing up, it can be observed that increase of solidity causes increase of power coefficient of the rotor, but this relationship is not proportional in the tested solidity range. Thus, one may conclude that the increase of the efficiency with the solidity raise can be true only up to a certain value, after which the further increase of solidity causes decay of $\mathrm{cp}$. The optimal rotor solidity depends on the blade geometry and atmospheric conditions (e.g. average wind speed) at a given location. For instance, at locations with lower annual average wind speed, the constructions with greater number of blades (consequently, due to higher solidity, having higher aerodynamic torque and lower cut-in speed) will be preferred.

Another issue is to calculate whether the greater power outcome gained e.g. from the 4-bladed SWT will counterbalance the higher costs of the production of greater number of blades. Therefore, the solidity raise may turn out to be a less profitable solution, than for example, increasing of the rotor swept area. 


\section{Summary and conclusions}

On the basis of the results presented in this paper, the following conclusions can be drawn:

- Greater number of blades, by increasing the solidity of the wind turbine rotor, allows to extract more kinetic energy from wind. Hence, the torque generated by the rotor raises and the cut-in wind speed decreases.

- The solidity raise causes the growth of the maximal $\mathrm{cp}$ value for a given rotor blades geometry. However, at some point the further solidity increase is expected to lead to the aerodynamic efficiency decrease.

- Higher aerodynamic efficiency of a 4-bladed rotor (comparing to its 3-bladed version) may indicate that there is still a big field for geometry improvements for the 3-bladed rotor.

- The increase of the rotor solidity results also in the growth of the form drag force. During the rotation, every blade experiences the wake disturbance caused by a preceding blade. Hence, the greater number of blades produces more considerable wake disturbance. As the tests revealed, this phenomenon becomes more significant at higher rotational speeds (TSR equal to 6.5 and higher).

- The numerical simulations confirmed the observations made in the experimental investigations and showed that the solidity change may result in greater aerodynamic efficiency in case of small wind turbines.

Summing up, tests in the wind tunnel and numerical simulations allowed to investigate the potential of modifying the SWT rotor performance by altering its solidity. The case study outcomes revealed the great potential of a 4-bladed rotor as well as the beneficial influence of blades with the chord length increased by $33 \%$, both yielding the growth of aerodynamic efficiency with respect to the baseline GUST rotor.

While the above observations give promising guidance for the rotor geometry amelioration, they should be benchmarked with further series of tests, especially to determine whether the same conclusions apply to the full-scale GUST wind turbine and what would be the influence of further modifications of solidity.

\section{References}

1. T. Wang, "A brief review on wind turbine aerodynamics", Theoretical and Applied Mechanics Letters, vol. 2, no. 6, p. 062001, 2012. Available: $10.1063 / 2.1206201$
2. H. Muhsen, W. Al-Kouz and W. Khan, "Small Wind Turbine Blade Design and Optimization", Symmetry, vol. 12 , no. 1, p. 18, 2019. Available: 10.3390/sym 12010018

3. A. Suresh and S. Rajakumar, "Design of small horizontal axis wind turbine for low wind speed rural applications", Materials Today: Proceedings, vol. 23, pp. 16-22, 2020. Available: 10.1016/j.matpr.2019.06.008

4. M. Lipian, I. Dobrev, M. Karczewski, F. Massouh and K. Jozwik, "Small wind turbine augmentation: Experimental investigations of shrouded- and twinrotor wind turbine systems", Energy, vol. 186, p. 115855, 2019.2 Available: 10.1016/j.energy.2019.115855

5. M. Kulak, M. Lipian and K. Zawadzki, "Investigation of performance of small wind turbine blades with winglets", International Journal of Numerical Methods for Heat \& Fluid Flow, vol. --, no. --, 2020. Available: 10.1108/hff-09-2019-0695

6. E. Hau and H. von Renouard, "Wind Turbines", 2006. Available: 10.1007/3-540-29284-5

7. R. Kumar and P. Baredar, "Solidity Study and its Effects on the Performance of A Small Scale Horizontal Axis Wind Turbine", Impending Power Demand and Innovative Energy Paths, pp. 290-297, 2012.

8. M. Hansen, Aerodynamics of Wind Turbines, 2nd edition. London: Taylor and Francis, 2013

9. T.Y. Chen and L.R. Liou, "Blockage corrections in wind tunnel tests of small horizontal-axis wind turbines", Experimental Thermal and Fluid Science, vol. 35, no. 3, pp. 565-569, 2011. Available: 10.1016/j.expthermflusci.2010.12.005

10. M. Lipian, M. Kulak and M. Stepien, "Fast Track Integration of Computational Methods with Experiments in Small Wind Turbine Development", Energies, vol. 12, no. 9, p. 1625, 2019. Available: 10.3390/en12091625

11. M. Drela, "XFOIL: An Analysis and Design System for Low Reynolds Number Airfoils. In Lecture Notes in Engineering: Low Reynolds Number Aerodynamics" Springer: New York, NY, USA, 1989 